\title{
Profiling the urinary microbiome in men with calcium-based kidney stones
}

\author{
Jing Xie, Jian-sheng Huang, Xiang-jiang Huang, Jun-ming Peng, Zhou Yu, Ye-qing Yuan, Ke-feng Xiao
}

and Ji-nan Guo*

\begin{abstract}
Background: The dogma that urine is sterile in healthy individuals has been overturned by recent studies applying molecular-based methods. Mounting evidences indicate that dysbiosis of the urinary microbiota is associated with several urological diseases. In this study, we aimed to investigate the urinary microbiome of male patients with calcium-based kidney stones and compare it with those of healthy individuals.

Results: The diversity of the urinary microbiota in kidney stone patients was significantly lower than that of healthy controls based on the Shannon and Simpson index ( $P<0.001$ for both indices). The urinary microbiota structure also significantly differed between kidney stone patients and healthy controls (ANOSIM, R $=0.11, P<0.001$ ). Differential representation of inflammation associated bacteria (e.g., Acinetobacter) and several enriched functional pathways were identified in the urine of kidney stones patients. Meanwhile, we found the species diversity, overall composition of microbiota and predicted functional pathways were similar between bladder urine and renal pelvis urine in kidney stone patients.
\end{abstract}

Conclusions: A marked dysbiosis of urinary microbiota in male patients with calcium-based kidney stones was observed, which may be helpful to interpret the association between bacteria and calcium-based kidney stones.

Keywords: Kidney stone, Calcium-based, Microbiome, Urine, Renal pelvis

\section{Background}

Nephrolithiasis is a common urological disorder worldwide, with a prevalence of $5-20 \%$ in different geographic regions and a recurrence rate of $50 \%$ at 10 years of follow-up [1]. The prevalence of nephrolithiasis has progressively risen during the last 30 years [2]. In China, the prevalence of nephrolithiasis in the periods of 19912000, 2001-2010, and 2011 to date was reported to be $5.95,8.86$ and $10.63 \%$, respectively [3]. Similar increases in prevalence exist in a variety of systemic diseases, such as diabetes, cardiovascular disease and metabolic syndrome [4-6]. Moreover, a recent population-based study demonstrated that multiple classes of oral antibiotics

\footnotetext{
* Correspondence: Guojn87@163.com

Department of Urology, Shenzhen People's Hospital, The Second Clinical Medical College of Jinan University, Shenzhen 518020, China
}

exposure is associated with increased odds of nephrolithiasis [7]. Interestingly, human microbiome could be affected by all these factors, indicating its potential role in the pathophysiology of nephrolithiasis.

The term human microbiome is defined as all genetic materials of micro-organisms existing in different regions of the body. In the past few years, the role of gut microbiome on urine oxalate excretion and kidney stone formation has been a hot issue. Early study identified distinct gut microbiome and enrichment of oxalate metabolizing bacterial species in nephrolithiasis patients [8]. Oxalobacter formigenes, an oxalate degradation bacterium, was reported to be negatively associated with urinary stones and reduce urinary oxalate excretion when administered orally as a probiotic [9]. Despite the promising preliminary data, further studies showed contradictions as to the colonization rate of O. formigenes,

C C The Author(s). 2020 Open Access This article is licensed under a Creative Commons Attribution 4.0 International License, which permits use, sharing, adaptation, distribution and reproduction in any medium or format, as long as you give appropriate credit to the original author(s) and the source, provide a link to the Creative Commons licence, and indicate if changes were made. The images or other third party material in this article are included in the article's Creative Commons licence, unless indicated otherwise in a credit line to the material. If material is not included in the article's Creative Commons licence and your intended use is not permitted by statutory regulation or exceeds the permitted use, you will need to obtain permission directly from the copyright holder. To view a copy of this licence, visit http://creativecommons.org/licenses/by/4.0/ The Creative Commons Public Domain Dedication waiver (http://creativecommons.org/publicdomain/zero/1.0/) applies to the data made available in this article, unless otherwise stated in a credit line to the data. 
ranging from 0 to $100 \%$ in kidney stone formers and $11-100 \%$ in individuals with no history of nephrolithiasis [10]. In addition, trials designed to degrade urinary oxalate with probiotics containing $O$. formigenes have been disappointing so far [11].

The urinary microbiome, identified in healthy individuals, is associated with several urologic diseases such as incontinence, genitourinary cancer and urinary tract infection [12, 13]. Early research observed that patients with non-struvite kidney stones often had positive urine cultures, indicating urinary microorganisms are associated with almost all types of kidney stones [14]. In a recent study, the urinary microbiome was showed to hold more relevant for urinary stones than the gut microbiome [15]. Collectively, these results suggest that urinary microbiome may be closely associated with nephrolithiasis. However, there have been limited studies to date that evaluate the association between urinary microbiome and calcium-based kidney stones [16]. In addition, whether the flora of bladder urine is distinct from that of renal pelvis urine also remains equivocal.

In the present study, we utilized $16 \mathrm{~S}$ rRNA gene sequencing to characterize the urinary microbiome potentially associated with calcium-based kidney stones. The aim of our research was to (1) determine if the microbiome of bladder urine is significantly different between kidney stone formers and healthy individuals; (2) determine if the microbiome of bladder urine is distinct from that of renal pelvis urine in nephrolithiasis patients. (3) predict functional pathways that significantly enriched in the urinary microbiome of kidney stone formers.

\section{Results}

\section{General characteristics of kidney stone patients and controls}

Urine samples were collected from a total of 43 subjects, and the demographic and clinical data was listed in Table 1. Age, gender, and body mass index showed no significant difference between kidney stone patients and healthy controls. Although comorbidities such as hypertension, diabetes and coronary artery disease were more common in kidney stone formers, they all did not reach statistical significance. The majority of renal stone patients were first onset $(20 / 22,90.9 \%)$ and only two patients were recurrent. All kidney stones were primarily calcium-based and composed of calcium oxalate, calcium phosphate, or a mixture of components. Pure calcium phosphate, uric acid, cystine or struvite stones were not identified. Antibiotics were given immediately after sample collection and no associated postoperative infections were identified in this study.
Table 1 Demographic and clinical data for the kidney stone formers and healthy subjects

\begin{tabular}{llll}
\hline & $\begin{array}{l}\text { Stone formers } \\
(\boldsymbol{n}=22)\end{array}$ & $\begin{array}{l}\text { Healthy controls } \\
(\boldsymbol{n}=21)\end{array}$ & $\boldsymbol{P}$ value \\
\hline Age, years & $46.9 \pm 10.1$ & $44.2 \pm 12.1$ & 0.435 \\
Female & 0 & 0 & 1.0 \\
$\mathrm{BMI}, \mathrm{kg} / \mathrm{m}^{2}$ & $24.3 \pm 3.28$ & $24.4 \pm 2.57$ & 0.876 \\
Comorbidities & & & \\
Hypertension & 5 & 2 & 0.410 \\
Diabetes & 2 & 0 & 0.488 \\
Coronary artery disease & 1 & 0 & 1.0 \\
Stone composition & & & $\mathrm{NA}$ \\
CaOx & 18 & NA & \\
CaOx + CaPhos & 3 & NA & \\
CaPhos & 0 & NA & \\
CaOx + Uric acid & 1 & NA & \\
Uric acid & 0 & NA & \\
\hline BMI Body mass index, CaOx Calcium oxalate, CaPhos Calcium phosphate
\end{tabular}

\section{Sequencing data and biodiversity of the urine microbiome}

In total, 5,906,796 clean reads were obtained from the 65 urine samples. The median number of reads in kidney stone patients was 94,966 and in healthy controls was 126,090 . The reads were classified into 928 unique operational taxonomic units (OTUs) at 97\% similarity level that were used for downstream analysis. We defined three groups according to the kidney stone status and specimen-type: HB represents bladder urine collected from healthy controls, KB represents bladder urine from kidney stone patients, while KP represents renal pelvis urine from kidney stone patients. The $\mathrm{HB}$ group showed the largest amount of OTUs, and there was substantial overlap in the OTUs composition among $\mathrm{HB}, \mathrm{KB}$ and KP groups (Fig. 1). Significant more OTUs were identified in the urine of healthy controls, with an average of 96 OTUs in HB group and 60 OTUs in KB group $(P=0.046)$.

For $\alpha$ - diversity, the values of Good's coverage index of all libraries were above $99 \%$. The $\alpha$-diversity indices, including observed species, chao 1 index, ACE index, Shannon diversity index, of the microbiota in HB group were all higher than those of KB group (Fig. 2). Moreover, significant differences were observed in Shannon diversity index and Simpson's diversity index between HB and KB groups $(P<0.001$ for both indices). The $\alpha-$ diversity of urinary microbiota between $K B$ and $K P$ group was also evaluated, and all indices showed no significant difference. For $\beta$-diversity, we applied unweighted and weighted principal coordinate analysis (PCoA) to display discrepancy among the three groups. It showed that KB and KP samples clustered closer in 


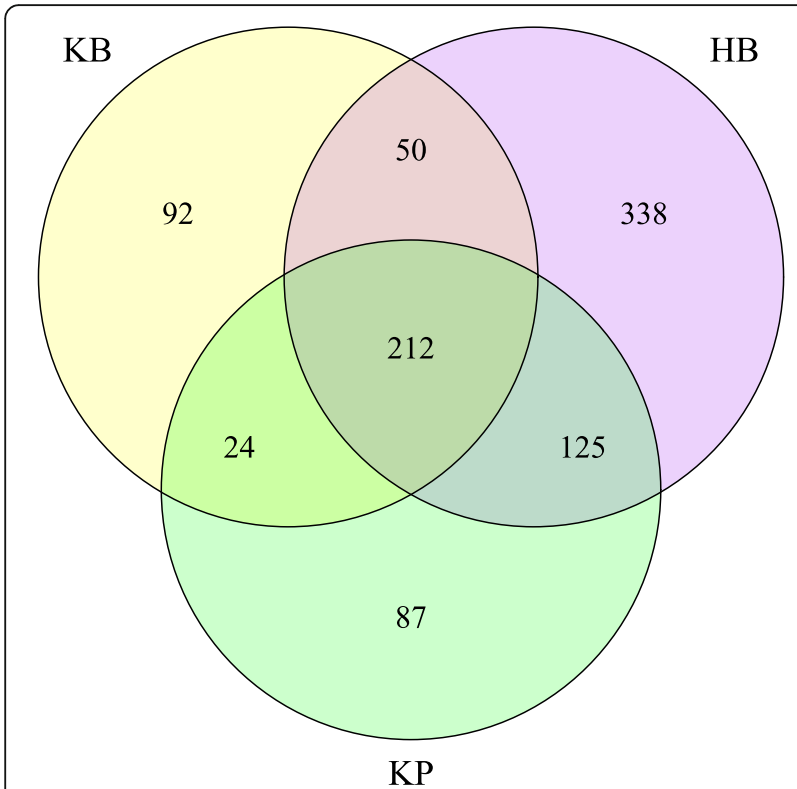

Fig. 1 Venn diagram of overlapping OTUs. A total of 928 OTUs were detected with 338 OTUs in HB samples only, 92 OTUs in KB samples only, 87 OTUs in KP samples only and 212 OTUs in all urine samples proximity to each other than HB samples (Fig. 3). We further performed analysis of similarities (ANOSIM), and found the urinary microbiota structure was significantly different between KB and HB groups (ANOSIM, $\mathrm{R}=0.11, P<0.001$ ), while the microbiota structure between $\mathrm{KB}$ and KP groups was similar (ANOSIM, $\mathrm{R}=$ $0.008, P=0.251)$.

\section{Taxonomic analysis of urine microbiota composition}

To identify the differentially represented taxa in kidney stone patients and controls, we compared the relative abundance of microbiota between $\mathrm{KB}$ and $\mathrm{HB}$ group at different taxonomic levels. At phylum level, a statistically significant difference was observed between these two groups in the average abundance of Bacteroidetes, Proteobacteria and Firmicutes. Namely, KB group showed a higher average representation of Proteobacteria $(51.8 \%$ vs $36.6 \%, p=0.01)$ and a lower average representation of Firmicutes $(29.3 \%$ vs $36.1 \%, p=0.02)$ and Bacteroidetes $(6.4 \%$ vs $19.4 \%, p<0.001)$. Significant abundance differences of numerous taxa were also noted between $\mathrm{KB}$ and $\mathrm{HB}$ groups at other taxonomic levels (Table 2). The relative abundance of Faecalibacterium
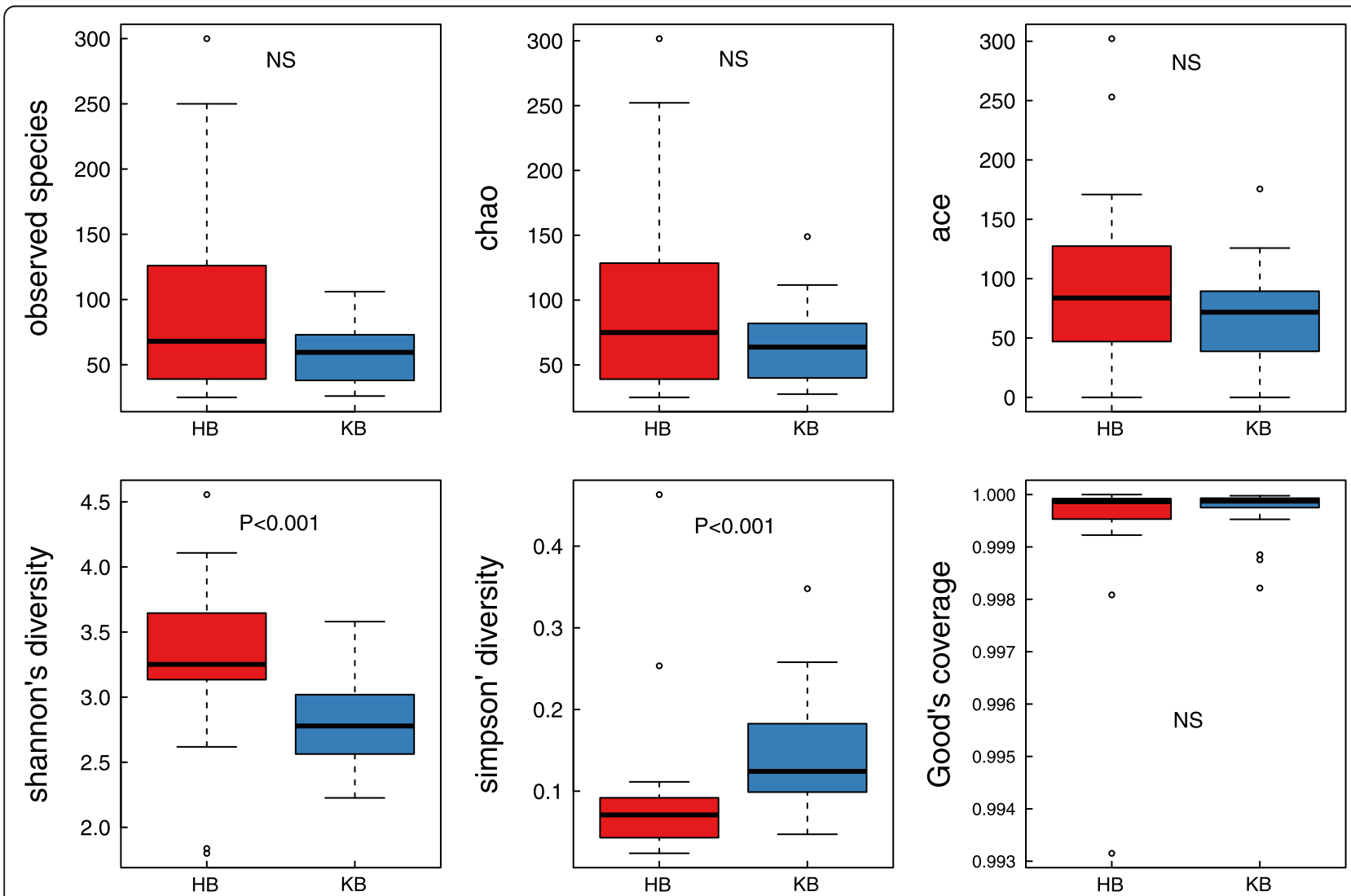

Fig. 2 Microbial a-diversity of urine samples. The a-diversity indices include observed species index, Chao 1 index, Ace index, Shannon index, Simpson index and Good's coverage index. Shannon diversity index and Simpson's diversity index were significantly different between HB and KB group 


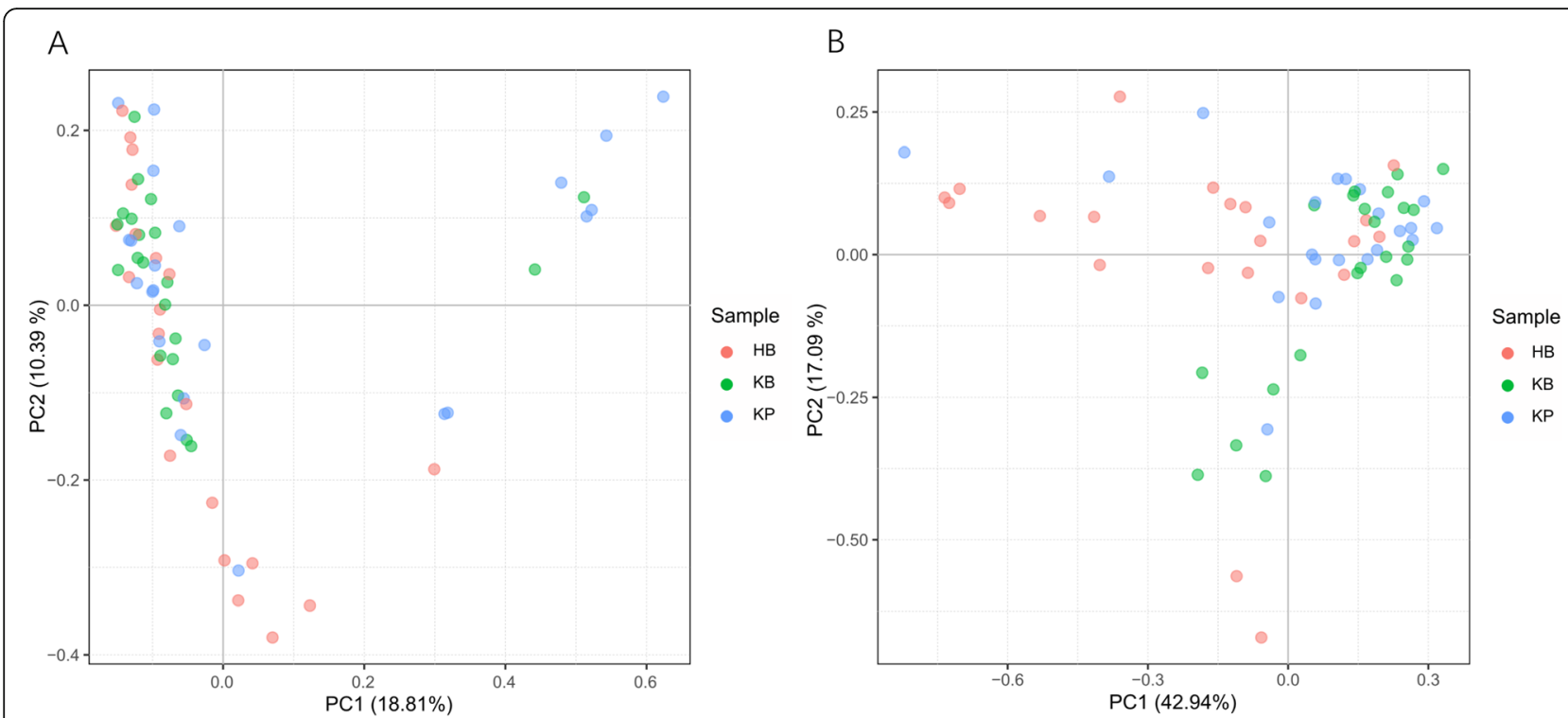

Fig. 3 Microbial $\beta$ - diversity analysis. PCOA plot of unweighted (a) and weighted (b) UniFrac metrics for HB (red dots), KB (green dots) and KP (blue dots) groups. Samples from KB and KP groups clustered closer in proximity to each other than HB samples

and Lactobacillus was also lower in KP and KB groups compared to $\mathrm{HB}$ group, although not statistically significant.

Of interest, we also analyzed the microbiota of paired bladder urine and renal pelvis urine collected from kidney stone patients. At phylum or class level, the overall bacterial compositions of KB and KP groups were quite similar (Fig. 4a-b). However, there were a few taxa differentially represented in these two groups at other taxonomic levels (Fig. 4c-e). A higher average representation of Anoxybacillus ( $1.2 \%$ vs $0.2 \%, p=0.01$ ) and lower average representation of Fusobacterium (0.6\% vs $1.3 \%, p=$ 0.02) was observed in KP group at genus level.

\section{Specific urinary genera associated with kidney stones}

To confirm the differentially abundant taxa in kidney stone patients and controls, we further applied LEfSe, a software using algorithm for high-dimensional biomarker discovery. Only taxa with logarithmic linear discriminant analysis (LDA) score more than 2.0 and $P<$ 0.05 in Wilcoxon test were considered differentially represented. LEfSe identified 31 discriminative features with significant different relative abundance among $\mathrm{HB}, \mathrm{KB}$ and KP groups (Fig. 5). The taxa at genus level that differentiated the three groups most were Prevotella in HB group, Acinetobacter in KB group and Anoxybacillus in KP group.

\section{Potential functional pathways associated with kidney stone}

Having observed a distinct urinary microbiota in kidney stone patients, we further evaluated whether the different bacterial community was associated with specific alterations involved in metabolic processes. The functional pathways of urinary microbiome in $\mathrm{HB}, \mathrm{KB}$ and KP samples were inferred using PICRUSt tool. Compared to HB group, the significantly enriched KEGG pathways in KB groups included proximal tubule bicarbonate reclamation, ion channels, linoleic acid metabolism and renin-angiotensin system (Supplementary Figure. 1). Meanwhile, the predicted KEGG pathways showed no significant difference between $K B$ and KP groups (Supplementary Figure. 2).

\section{Discussion}

In the present study, we utilized $16 \mathrm{~S}$ rRNA gene sequencing to explore the urinary microbiome in male calciumbased kidney stone formers and age-matched healthy individuals. The noted improvement in our study was the strict inclusion criteria, aiming to control various confounding factors. Our results showed significantly reduced species diversity and altered microbial profile in the urine of kidney stone patients compared to controls. Several differentially represented taxa and functional pathways were found in HB and KB groups. In addition, we found the overall bacterial composition and predicted functional pathways of bladder urine was similar to that of renal pelvis urine in kidney stone patients.

Urolithiasis is a common urological disorder with diverse pathologies and the factors contributing to the increasing prevalence are currently unknown. In the previous literature, the mechanisms that urea-producing bacteria promoting struvite stone formation have been well documented. However, the potential mechanism 
Table 2 Comparison of average relative abundance of bladder urine microbiome in kidney stone patients and healthy subjects at different taxonomic levels

\begin{tabular}{|c|c|c|c|c|c|c|}
\hline \multirow[t]{2}{*}{ Taxa } & & \multicolumn{3}{|c|}{ Average abundance (\%) } & \multicolumn{2}{|c|}{ Prevalence (\%) } \\
\hline & & $\boldsymbol{P}$ value & $\mathrm{HB}$ & KB & $\overline{\mathrm{HB}}$ & KB \\
\hline \multirow[t]{3}{*}{ Phylum } & Bacteroidetes & $<0.001$ & 19.362 & 6.402 & 100 & 100 \\
\hline & Proteobacteria & 0.013 & 36.641 & 51.797 & 100 & 100 \\
\hline & Firmicutes & 0.024 & 36.114 & 29.318 & 100 & 100 \\
\hline \multirow[t]{7}{*}{ Family } & Moraxellaceae & $<0.001$ & 11.960 & 32.655 & 100 & 100 \\
\hline & Prevotellaceae & 0.001 & 8.992 & 1.572 & 100 & 73 \\
\hline & Odoribacteraceae & 0.008 & 0.110 & 0.000 & 29 & 0 \\
\hline & Fusobacteriaceae & 0.012 & 0.374 & 1.303 & 43 & 73 \\
\hline & Porphyromonadaceae & 0.014 & 0.754 & 0.341 & 67 & 27 \\
\hline & Enterococcaceae & 0.041 & 0.494 & 0.913 & 57 & 73 \\
\hline & Planococcaceae & 0.047 & 0.132 & 0.487 & 38 & 59 \\
\hline \multirow[t]{9}{*}{ Genus } & Acinetobacter & $<0.001$ & 10.996 & 31.383 & 100 & 100 \\
\hline & Prevotella & 0.001 & 9.377 & 1.628 & 100 & 73 \\
\hline & Desulfovibrio & 0.008 & 0.178 & 0.000 & 29 & 0 \\
\hline & Eubacterium & 0.008 & 0.039 & 0.000 & 29 & 0 \\
\hline & Odoribacter & 0.008 & 0.083 & 0.000 & 29 & 0 \\
\hline & Fusobacterium & 0.012 & 0.374 & 1.297 & 43 & 73 \\
\hline & Parabacteroides & 0.017 & 0.540 & 0.252 & 57 & 23 \\
\hline & Lysinibacillus & 0.020 & 0.069 & 0.468 & 29 & 55 \\
\hline & Oscillospira & 0.039 & 0.626 & 0.019 & 38 & 14 \\
\hline \multirow[t]{12}{*}{ Species } & Acinetobacter johnsonii & $<0.001$ & 7.310 & 26.039 & 100 & 100 \\
\hline & Prevotella copri & $<0.001$ & 7.272 & 0.431 & 86 & 59 \\
\hline & Prevotella stercorea & 0.002 & 0.422 & 0.031 & 57 & 14 \\
\hline & Clostridium sartagoforme & 0.004 & 0.099 & 0.000 & 33 & 0 \\
\hline & Bacteroides barnesiae & 0.008 & 0.369 & 0.000 & 29 & 0 \\
\hline & Eubacterium biforme & 0.008 & 0.031 & 0.000 & 29 & 0 \\
\hline & Lysinibacillus boronitolerans & 0.020 & 0.069 & 0.468 & 29 & 55 \\
\hline & Bacteroides ovatus & 0.021 & 1.084 & 0.212 & 57 & 23 \\
\hline & Parabacteroides distasonis & 0.030 & 0.337 & 0.047 & 43 & 14 \\
\hline & Bacteroides fragilis & 0.033 & 0.368 & 0.070 & 43 & 14 \\
\hline & Bacteroides plebeius & 0.037 & 1.473 & 1.876 & 71 & 91 \\
\hline & Veillonella parvula & 0.047 & 0.364 & 0.213 & 38 & 9 \\
\hline
\end{tabular}

that bacteria contribute to calcium-based stone, the vast majority of kidney stones, remains obscure. One hypothesis is that bacteria adhere to crystal and promote its growth and aggregation. This is supported by findings that bacteria such as Enterobacteriaceae selectively aggregated to oxalate calcium crystal and increased the number of aggregations [17]. Similar crystal aggregation ability was observed in Staphylococcus and Streptococcus species in vitro [18]. Another possibility is that bacteria may alter urine supersaturation via production of citrate lyase, which decreases the urine citrate levels and lead to crystal formation [19]. Lastly, bacteria may induce an inflammatory response and the release of proinflammatory proteins, which form the stone matrix inner core and progress from crystal to stone [20].

The main finding of this study was that we demonstrated distinct urinary microbiota in kidney stone patients compared to healthy subjects. Our results showed that nephrolithiasis patients had significant lower species diversity in urine. According to previous literature, decreased microbiota diversity was related to inflammation and implicated in diseases such as obesity and type II diabetes [21]. Moreover, we found several bacterial taxa associated with inflammation were overrepresented or 

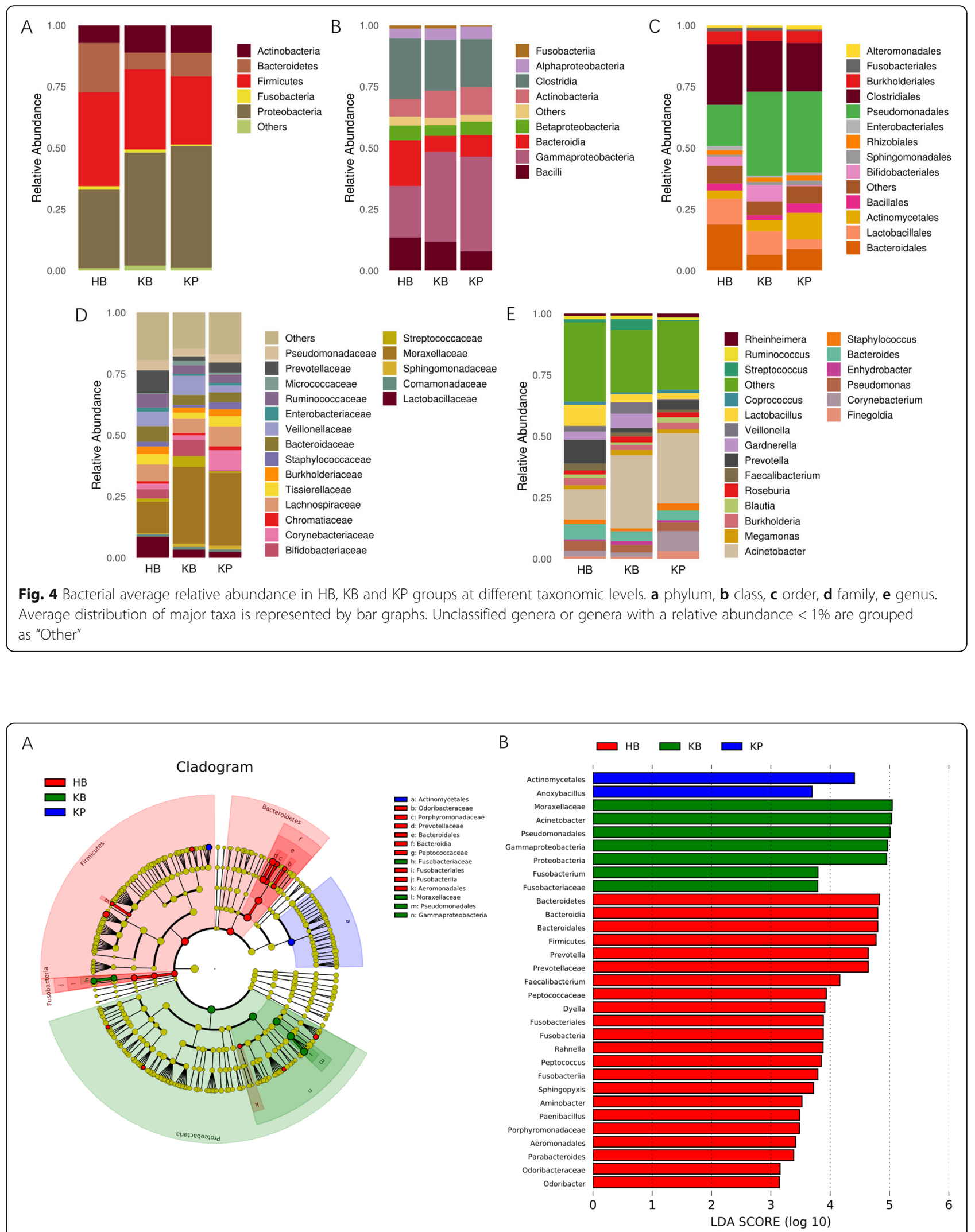

Fig. 5 Cladogram (a) and LEfSe analyses (b) of microbiomes among HB (red), KB (green) and KP (blue) groups. Taxa in graph were with LDA score threshold $>2.0$ and statistically significant $(p<0.05)$ 
underrepresented in the urine of kidney stone patients. The most differentially represented taxa at genus level were Acinetobacter in kidney stone patients, and Prevotella in healthy controls. As opportunistic pathogens, Acinetobacter are associated with urinary tract infection in individuals with underlying medical risk factors, such as diabetes mellitus and immunosuppression [22]. Interestingly, the abundance of Acinetobacter was showed to be higher in the faeces of nephrolithiasis patients and the urine of bladder cancer patients compared to controls, although its association with plasma trace elements or bladder cancer recurrence/progression had not been identified [23, 24]. Prevotella are classically considered as commensal bacteria and known to colonize the gastrointestinal tract, vaginal tract and urinary tract. It could synthetize short-chain fatty acids, which were able to protect against inflammation in acute kidney injury [25]. The decreased level of Prevotella favours inflammatory processes and has been implicated in several pathological conditions, including type 2 diabetes, diabetic nephropathy and chronic prostatitis [26, 27].

In summary, our results revealed significantly decreased species diversity, enrichments of proinflammatory bacteria and underrepresentation of antiinflammatory taxa in the urinary microbiota of kidney stone patients. Similar trends were showed by Zampini and colleagues that a long-term shift in urinary tract microbiome may increase the risk for urinary stones, although not excluding subjects using antibiotics [15]. We also predicted several functional pathways which were significantly enriched in the urinary microbiome of kidney stone patients compared to healthy controls. Among these pathways, ion channels are key regulators of the cell membrane and have been demonstrated as an entrance gate in bacteria-host interactions [28]. In an infection-induced urolithiasis rat model, the activities of calcium related ion channels were reported to be influenced by bacterial infection, and correlated with chronic inflammation of the kidney along with rapid aggregation of stones [29]. Moreover, transient receptor potential vanilloid 5 (TRPV5), a member of the transient receptor potential family of ion channels, has also been proved to be closely associated with urinary stone formation [30]. In the present study, our results revealed enriched ion channels pathway in the urine of nephrolithiasis patients, but its specific role in kidney stone formation still remains unclear and needs further investigation. Nevertheless, it is reasonable to speculate that bacteria might influence the formation of calcium-based stones via modulation of inflammatory processes.

Another important finding in this study was the similarity of overall bacterial composition between bladder urine and renal pelvis urine in kidney stone patients. Traditionally, bacteria are considered to access the upper urinary tract under certain conditions, such as urinary reflux or bacteria translocation in severe systemic disease. However, a preliminary study showed bacteria could be detected in the upper tract urine of kidney stone patients without urinary tract infections [17]. Due to the small sample size, the author did not compare the microbiota between bladder urine and upper tract urine. In a recent study, Dornbier and colleagues found that there was no significant difference in the microbial composition of bladder urine and upper tract urine in urinary stone patients [16]. It is worth noting that ureteral stents were placed in the majority of patients $(50 / 52$, 96.1\%) in that study, which may potentially influence the urinary microbiota. In the present study, we found that the species diversity and overall composition of microbiota was similar between KB and KP groups, after excluding confounding factors such as antibiotic use and ureteral stent placement. In addition, our PICRUSt results showed no significant difference with regard to the predicted functional pathways between $\mathrm{KB}$ and $\mathrm{KP}$ groups. Meanwhile, we also noted that there were a few taxa (e.g., Anoxybacillus) differentially represented in KP group, remaining an area for future research.

Some limitations should be noted when interpreting our results. First, all participants were Chinese and the sample size is relatively small, limiting generalizability and comparison of stone subtypes. Further largescale studies are necessary to investigate the urinary tract microbiota across ethnicity and stone type. Second, this study did not include female subjects, mainly due to their lower morbidity of kidney stones and higher positive rate of urine routine tests. In the future, we will conduct a more comprehensive research after recruiting adequate females in line with our inclusion criteria. Additionally, the association of risk factors for lithogenesis in urine and urine microbiota was not evaluated, because the vast majority of kidney stone patients were first onset and $24 \mathrm{~h}$ urine analyses were not performed. Finally, like most metagenomic studies, we cannot comment as to whether altered urinary microbiota in kidney stone patients was a contributor or the result of kidney stone formation. All these questions will certainly be the focus of future research.

\section{Conclusions}

In conclusion, our study revealed distinct urinary microbiota in male kidney stone patients compared to healthy individuals, and similar microbiota between bladder urine and renal pelvis urine. Several predicted functional pathways and bacteria associated with inflammation were found to differentially represent in the urinary tract of kidney stone patients. We speculated that bacteria might influence the formation of calcium-based kidney stones via modulation of inflammatory processes. Our 
findings may provide useful information to interpret the association between bacteria and calcium-based kidney stones.

\section{Methods}

\section{Recruitment of participants}

We recruited a total of 43 adult males, including 22 kidney stone formers and 21 age-matched healthy volunteers at Shenzhen People's Hospital. All nephrolithiasis patients were diagnosed by ultrasonography, abdominal plain film, intravenous pyelography or computed tomography, and kidney stones were confirmed during endoscopic surgery. The chemical composition of surgically removed stones was analyzed by infrared spectroscopy.

In order to control the confounding factors that might affect urinary microbiome, we set strict exclusion criteria. For healthy controls, exclusion criteria included personal history of urinary stones, episodes of renal colic or imaging confirmed urinary stones. All healthy controls underwent ultrasonography to confirm the lack of asymptomatic renal calculus. For kidney stone patients, exclusion criteria included struvite stones, concurrent ureteral calculus, moderate to severe hydronephrosis, and ureteral stent or catheter placement before sample collection. Excluded from both groups were subjects using antibiotic within 30 days, with urinary tract infections or positive urine culture, congenital abnormalities of the urinary tract, history of major urological surgery, diabetes with poorly controlled glucose, autoimmune disease, chronic kidney disease with blood creatinine > $1.4 \mathrm{mg} / \mathrm{dL}$ and age $(<20$ years or $>70$ years old).

\section{Sample collection and processing}

Bladder urine samples were obtained by transurethral catheterization from all participants. For nephrolithiasis patients, paired renal pelvis urine samples were collected on the side of kidney stones via ureteral catheter using aseptic technique, prior to surgery or ureteral stent insertion. Before the renal pelvis urine collection, the bladder was voided by catheter with an attempt to control the mixture of bladder urine. All samples were collected prior to antibiotic use and stored in sterile containers at $-80^{\circ} \mathrm{C}$ within $1 \mathrm{~h}$ from collection. The volume of each urine sample was approximately $12 \mathrm{ml}$ and the time for renal pelvis urine collection was approximately $10 \mathrm{~min}$.

\section{DNA extraction and 16S rRNA amplicon sequencing}

Prior to DNA extraction, all samples were centrifuged $12,000 \mathrm{~g}$ for $10 \mathrm{~min}$ at $4{ }^{\circ} \mathrm{C}$. Pellets were re-suspended and mixed with DNA-free phosphate buffered saline. Genomic DNA was extracted from all samples using DNeasy PowerWater Kit (MoBio, USA). Integrity of DNA was verified with agarose gel electrophoresis and the DNA concentration was quantified by Qubit ${ }^{\circledR} 2.0$
Fluorometer (Life Technologies, USA). All DNA extractions were stored at $-20^{\circ} \mathrm{C}$ until further processing.

The V3-V4 region of the $16 \mathrm{~S}$ rRNA gene was amplified by polymerase chain reaction with primers shown as follows: V3-V4-341F: 5' - CCTACGGGNGGCWGCAG3' and 907R: 5'-TACNVGGGTATCTAATCC-3'. Polymerase chain reaction was performed using the following conditions: $3 \mathrm{~min}$ denaturation at $98^{\circ} \mathrm{C} ; 30$ cycles of denaturation at $98^{\circ} \mathrm{C}$ for $45 \mathrm{~s}$, annealing at $55^{\circ} \mathrm{C}$ for 45 $\mathrm{s}$, elongation at $72{ }^{\circ} \mathrm{C}$ for $45 \mathrm{~s}$; and final extension at $72{ }^{\circ} \mathrm{C}$ for $7 \mathrm{~min}$. The amplicons were purified by the AMPure beads (Axygen, USA), and barcoded libraries were sequenced on the Illumina Hiseq2500 platform. Sterile phosphate buffered saline with and without bullet blender beads were used as negative controls during processing.

\section{Bioinformatic analysis}

Raw sequencing data was pre-processed to eliminate low-quality reads and adapter pollution by using Mothur [31]. Clean reads were merged to tags using FLASH software [32] and the latter were assigned to OTUs based on $97 \%$ sequence similarity using USEARCH [33]. The representative sequences of each OTU were taxonomically classified by RDP Classifier [34] based on the Greengenes database [35]. Sequences associated with chimeras, chloroplasts and mitochondria were removed prior to downstream analyses [36]. Due to the low biomass nature of urine samples, the threshold for sequence positivity was conservatively set at a cutoff of 2000 sequence reads [16].

For $\alpha$-diversity, observed species, chao 1 index, ACE index, Shannon index, Simpson index and Goodcoverage index were calculated by Mothur. For $\beta$-diversity, both unweighted and weighted UniFrac distances were conducted using QIIME and shown by the PCoA [37]. Analysis was performed to find biomarkers differentially represented among the sample groups by LEfSe software [38]. The threshold on the logarithmic LDA score for discriminative features was 2.0. The functional pathways of bacterial community were inferred by utilizing PICRUSt algorithm [39]. In brief, the OTU table was imported to PICRUSt software and functional predictions were performed using Kyoto Encyclopedia of Genes and Genomes (KEGG) orthology.

\section{Statistical analyses}

Statistical analyses were performed with the SPSS (version 21.0) and $\mathrm{R}$ software (version 3.4.1), considering $p$ values $<0.05$ as statistically significant. Clinical categorical variables were compared using Pearson's chi-square test or Fisher's Exact Test, while continuous variables were analyzed via a student's t test. Age and body mass index were expressed as mean \pm standard deviation. For 
$\alpha$-diversity and taxonomic analysis, Wilcoxon rank-sum test or Kruskal-Wallis test were performed with $\mathrm{R}$ software. For $\beta$-diversity, statistical comparisons of weighted UniFrac distances were conducted by ANOSIM using the vegan package of $\mathrm{R}$ software.

\section{Supplementary information}

Supplementary information accompanies this paper at https://doi.org/10. 1186/s12866-020-01734-6.

Additional file 1: Figure S1. Microbial pathways that were significantly differentially enriched between $\mathrm{HB}$ and KB groups.

Additional file 2: Figure S2. Predicted microbial pathways were not significantly differentially represented between KB and KP groups.

\section{Abbreviations}

ANOSIM: Analysis of similarities; HB: Bladder urine collected from healthy individuals; KB: Bladder urine collected from nephrolithiasis patients; KP: Renal pelvis urine collected from nephrolithiasis patients; LDA: Linear discriminant analysis; OTU: Operational taxonomic unit; PCOA: Principal coordinate analysis

\section{Acknowledgements}

Not applicable.

\section{Authors' contributions}

All authors participated in the conception and design of the study; conceived and drafted the manuscript: JX, JSH, XJH; performed the experiments: JX, JMP, ZY; collected the basic patient information, clinical indicators: JX, JSH, YQY; analyzed the data: JX, KFX, JNG; revised the paper: KFX and JNG. All authors read and approved the final manuscript.

\section{Funding}

This study was supported by The Science and Technology Foundation of Shenzhen (grant no. JCYJ20170307095620828). Funding bodies had no role in study design, collected data, analysis, or writing.

\section{Availability of data and materials}

All data generated or analysed during this study are included in this published article and its supplementary information files. The datasets used and/or analysed during the current study are also available from the corresponding author on reasonable request.

\section{Ethics approval and consent to participate}

The study was approved by the local Ethics Committee of Second Clinical Medical College of Jinan University. All subjects provided written informed consent before they participated in this study.

\section{Consent for publication}

Not applicable.

\section{Competing interests}

The authors declare that they have no competing interests.

Received: 21 December 2019 Accepted: 24 February 2020

Published online: 28 February 2020

\section{References}

1. Türk C, Skolarikos A, Neisius A, Petř́k A, Seitz C, Thomas K. Guidelines on urolithiasis. European Association of Urology. 2019. https://uroweb.org/ guideline/urolithiasis/. Accessed 11 Dec 2019.

2. Scales CD Jr, Smith AC, Hanley JM, Saigal CS. Urologic Diseases in America Project Prevalence of kidney stones in the United States. Eur Urol. 2012;62: 160-5.

3. Wang W, Fan J, Huang G, Li J, Zhu X, Tian Y, et al. Prevalence of kidney stones in mainland China: a systematic review. Sci Rep. 2017. https://dol. org/10.1038/srep41630
4. Menke A, Casagrande S, Geiss L, Cowie CC. Prevalence of and trends in diabetes among adults in the United States, 1988-2012. JAMA. 2015;314: 1021-9.

5. Schnabel RB, Yin X, Gona P, Larson MG, Beiser AS, McManus DD, et al. 50 year trends in atrial fibrillation prevalence, incidence, risk factors, and mortality in the Framingham heart study: a cohort study. Lancet. 2015;386: 154-62.

6. Ng M, Fleming T, Robinson M, Thomson B, Graetz N, Margono C, et al Global, regional, and national prevalence of overweight and obesity in children and adults during 1980-2013: a systematic analysis for the global burden of disease study 2013. Lancet. 2014;384:766-81.

7. Tasian GE, Jemielita T, Goldfarb DS, Copelovitch L, Gerber JS, Wu Q, et al. Oral antibiotic exposure and kidney stone disease. J Am Soc Nephrol. 2018; 29:1731-40

8. Suryavanshi MV, Bhute SS, Jadhav SD, Bhatia MS, Gune RP, Shouche YS Hyperoxaluria leads to dysbiosis and drives selective enrichment of oxalate metabolizing bacterial species in recurrent kidney stone endures. Sci Rep. 2016. https://doi.org/10.1038/srep34712.

9. Siener R, Bangen U, Sidhu H, Hönow R, von Unruh G, Hesse A. The role of Oxalobacter formigenes colonization in calcium oxalate stone disease. Kidney Int. 2013:83:1144-9.

10. Batagello CA, Monga M, Miller AW. Calcium oxalate urolithiasis: a case of missing microbes? J Endourol. 2018;32:995-1005.

11. Lieske JC. Probiotics for prevention of urinary stones. Ann Transl Med. 2017; 5:29.

12. Li JKM, Chiu PKF, Ng CF. The impact of microbiome in urological diseases: a systematic review. Int Urol Nephrol. 2019;51:1677-97.

13. Shrestha E, White JR, Yu SH, Kulac I, Ertunc O, De Marzo AM, et al. Profiling the urinary microbiome in men with positive versus negative biopsies for prostate cancer. J Urol. 2018:199:161-71.

14. Tavichakorntrakool R, Prasongwattana V, Sungkeeree S, Saisud P, Sribenjalux P. Pimratana $C$, et al. Extensive characterizations of bacteria isolated from catheterized urine and stone matrices in patients with nephrolithiasis. Nephrol Dial Transplant. 2012;27:4125-30

15. Zampini A, Nguyen AH, Rose E, Monga M, Miller AW. Defining dysbiosis in patients with urolithiasis. Sci Rep. 2019. https://doi.org/10.1038/s41598-01941977-6.

16. Dornbier RA, Bajic P, Van Kuiken M, Jardaneh A, Lin H, Gao X, et al. The microbiome of calcium-based urinary stones. Urolithiasis. 2019. https://doi. org/10.1007/s00240-019-01146-w.

17. Barr-Beare E, Saxena V, Hilt EE, Thomas-White K, Schober M, Li B, et al. The interaction between enterobacteriaceae and calcium oxalate deposits. PLoS One. 2015. https://doi.org/10.1371/journal.pone.0139575

18. Chutipongtanate S, Sutthimethakorn S, Chiangjong W, Thongboonkerd V. Bacteria can promote calcium oxalate crystal growth and aggregation. J Biol Inorg Chem. 2013;18:299-308.

19. De Ferrari ME, Macaluso M, Brunati C, Pozzoli R, Colussi G. Hypocitraturia and Ureaplasma urealyticum urinary tract infection in patients with idiopathic calcium nephrolithiasis. Nephrol Dial Transplant. 1996;11:1185.

20. Djojodimedjo T, Soebadi DM. Soetjipto. Escherichia coli infections induces mucosal damage and expression of proteins promoting urinary stone formation. Urolithiasis. 2013:41:295-301.

21. Pascale A, Marchesi N, Govoni S, Coppola A, Gazzaruso C. The role of gut microbiota in obesity, diabetes mellitus, and effect of metformin: new insights into old diseases. Curr Opin Pharmacol. 2019;49:1-5.

22. Elhosseiny NM, Attia AS. Acinetobacter: an emerging pathogen with a versatile secretome. Emerg Microbes Infect. 2018;7:33.

23. Tang $R$, Jiang $Y$, Tan $A$, Ye J, Xian X, Xie $Y$, et al. 16S rRNA gene sequencing reveals altered composition of gut microbiota in individuals with kidney stones. Urolithiasis. 2018;46:503-14.

24. Wu P, Zhang G, Zhao J, Chen J, Chen Y, Huang W, et al. Profiling the urinary microbiota in male patients with bladder cancer in China. Front Cell Infect Microbiol. 2018:8:167.

25. Andrade-Oliveira V, Amano MT, Correa-Costa M, Castoldi A, Felizardo RJ, de Almeida DC, et al. Gut bacteria products prevent AKI induced by ischemiareperfusion. J Am Soc Nephrol. 2015;26:1877-88

26. Tao S, Li L, Li L, Liu Y, Ren Q, Shi M, et al. Understanding the gut-kidney axis among biopsy-proven diabetic nephropathy, type 2 diabetes mellitus and healthy controls: an analysis of the gut microbiota composition. Acta Diabetol. 2019;56:581-92. 
27. Shoskes DA, Wang H, Polackwich AS, Tucky B, Altemus J, Eng C. Analysis of gut microbiome reveals significant differences between men with chronic prostatitis/chronic pelvic pain syndrome and controls. J Urol. 2016;196:43541.

28. Feske S, Wulff H, Skolnik EY. Ion channels in innate and adaptive immunity. Annu Rev Immunol. 2015;33:291-353.

29. Cherng JH, Hsu YJ, Liu CC, Tang SH, Sartika D, Chang SJ, et al. Activities of Ca2+-related ion channels during the formation of kidney stones in an infection-induced urolithiasis rat model. Am J Physiol Ren Physiol. 2019;317: F1342-9.

30. van der Wijst J, van Goor MK, Schreuder MF, Hoenderop JG. TRPV5 in renal tubular calcium handling and its potential relevance for nephrolithiasis. Kidney Int. 2019;96:1283-91.

31. Schloss PD, Westcott SL, Ryabin T, Hall JR, Hartmann M, Hollister EB, et al. Introducing mothur: open-source, platform-independent, communitysupported software for describing and comparing microbial communities. Appl Environ Microbiol. 2009;75:7537-41.

32. Magoč T, Salzberg SL. FLASH: fast length adjustment of short reads to improve genome assemblies. Bioinformatics. 2011;27:2957-63.

33. Edgar RC. UPARSE: highly accurate OTU sequences from microbial amplicon reads. Nat Methods. 2013;10:996-8.

34. Wang Q, Garrity GM, Tiedje JM, Cole JR. Naïve Bayesian classifier for rapid assignment of rRNA sequences into the new bacterial taxonomy. Appl Environ Microbiol. 2007;73:5261-7.

35. DeSantis TZ, Hugenholtz P, Larsen N, Rojas M, Brodie EL, Keller K, et al. Greengenes, a chimera-checked 16S rRNA gene database and workbench compatible with ARB. Appl Environ Microbiol. 2006;72:5069-72.

36. Miller AW, Dale C, Dearing MD. The induction of oxalate metabolism in vivo is more effective with functional microbial communities than with functional microbial species. mSystems. 2017. https://doi.org/10.1128/ mSystems.00088-17.

37. Tang R, Wei Y, Li Y, Chen W, Chen H, Wang Q, et al. Gut microbial profile is altered in primary biliary cholangitis and partially restored after UDCA therapy. Gut. 2018;67:534-41.

38. Segata N, lzard J, Waldron L, Gevers D, Miropolsky L, Garrett WS, et al. Metagenomic biomarker discovery and explanation. Genome Biol. 2011. https://doi.org/10.1186/gb-2011-12-6-r60.

39. Langille MG, Zaneveld J, Caporaso JG, McDonald D, Knights D, Reyes JA, et al. Predictive functional profiling of microbial communities using 165 rRNA marker gene sequences. Nat Biotechnol. 2013;31:814-21.

\section{Publisher's Note}

Springer Nature remains neutral with regard to jurisdictional claims in published maps and institutional affiliations.

Ready to submit your research? Choose BMC and benefit from:

- fast, convenient online submission

- thorough peer review by experienced researchers in your field

- rapid publication on acceptance

- support for research data, including large and complex data types

- gold Open Access which fosters wider collaboration and increased citations

- maximum visibility for your research: over $100 \mathrm{M}$ website views per year

At $\mathrm{BMC}$, research is always in progress.

Learn more biomedcentral.com/submissions 